\title{
Producción de la glicoproteína C recombinante de alfaherpesvirus equino 1 y evaluación de la respuesta inmune inducida en ratones
}

\begin{abstract}
Production of recombinant glycoprotein $C$ from Equid alphaherpesvirus 1 and evaluation of the immune response induced in mice
\end{abstract}

Fuentealba, Nadia Analía; Bravi, María Emilia; Panei, Carlos Javier; Zanuzzi, Carolina Natalia; Sguazza, Guillermo Hernán; Corva, Santiago Gerardo; Pecoraro, Marcelo Ricardo; Galosi, Cecilia Mónica

\section{Fuentealba, Nadia Analía}

nadiafuentealba@fcv.unlp.edu.ar

Cátedra y Laboratorio de Virología (LAVIR).

Facultad de Ciencias Veterinarias, Universidad

Nacional de La Plata. Consejo Nacional de

Investigaciones Científicas y Técnicas

(CONICET-CCT-La Plata), Argentina

\section{(iD) Bravi, María Emilia}

Cátedra y Laboratorio de Virología (LAVIR).

Facultad de Ciencias Veterinarias, Universidad

Nacional de La Plata. Consejo Nacional de

Investigaciones Científicas y Técnicas

(CONICET-CCT-La Plata), Argentina

\section{(D) Panei, Carlos Javier}

Cátedra y Laboratorio de Virología (LAVIR).

Facultad de Ciencias Veterinarias, Universidad

Nacional de La Plata. Consejo Nacional de

Investigaciones Científicas y Técnicas

(CONICET-CCT-La Plata), Argentina

\section{(iD) Zanuzzi, Carolina Natalia}

Cátedra de Histología y Embriología, Facultad de Ciencias Veterinarias, Universidad Nacional de La Plata. Consejo Nacional de Investigaciones

Científicas y Técnicas (CONICET-CCT-La Plata), Argentina

Sguazza, Guillermo Hernán

Cátedra y Laboratorio de Virología (LAVIR).

Facultad de Ciencias Veterinarias, Universidad

Nacional de La Plata, Argentina

Corva, Santiago Gerardo

Cátedra de Bioestadística. Facultad de Ciencias

Veterinarias, Universidad Nacional de La Plata, Argentina

\section{Pecoraro, Marcelo Ricardo}

Resumen: El alfaherpesvirus equino 1 (EHV-1) produce enfermedad respiratoria, abortos, muerte perinatal y desórdenes neurológicos en miembros de la familia Equidae. Las glicoproteínas de la envoltura viral son los antígenos inmunodominantes que generan respuesta inmune en animales infectados. La infección natural y las vacunas disponibles proporcionan protección parcial y de corta duración contra la reinfección. En este trabajo se expresó la glicoproteína $\mathrm{C}$ viral utilizando un baculovirus recombinante y se analizó la capacidad de inducir inmunidad protectora en ratones $\mathrm{BALB} / \mathrm{c}$ al ser desafiados con el virus. Se evaluaron diferentes rutas de administración y distintos adyuvantes. La glicoproteína C indujo una respuesta IgG específica en suero; sin embargo, no logró evitar la entrada del virus al pulmón ni la aparición de signos clínicos, los cuales fueron variables entre los diferentes grupos de ratones. En todos los grupos inmunizados se retrasó la entrada del virus al pulmón y, cuando se utilizaron adyuvantes, el título viral fue significativamente menor que en el grupo control, así como en el grupo inmunizado por vía intranasal. Se concluye que la glicoproteína $\mathrm{C}$ producida indujo protección parcial frente al desafío viral según el adyuvante y la vía deinmunización.

Palabras clave: alfaherpesvirus equino 1, glicoproteína $\mathrm{C}$, respuesta inmune, ratón BALB/c.
Abstract: Equid alphaherpesvirus 1 (EHV-1) causes respiratory disease, abortions, perinatal death and neurological disorders in horses. Envelope glycoproteins are the most immunodominant antigens in infected animals. Natural infection and available vaccines provide partial and short-term protection against reinfection. In this study, EHV-1 glycoprotein C was expressed with a recombinant baculovirus and the ability to induce protective immunity in BALB/c mice challenged with the virus was analyzed. Different routes of administration and adjuvants were evaluated. 
Cátedra y Laboratorio de Virología (LAVIR). Facultad de Ciencias Veterinarias, Universidad Nacional de La Plata, Argentina

\section{(D) Galosi, Cecilia Mónica}

Cátedra y Laboratorio de Virología (LAVIR).

Facultad de Ciencias Veterinarias, Universidad Nacional de La Plata. Comisión de Investigaciones Científicas de la Provincia de Buenos Aires, Argentina

\section{ANALECTA VETERINARIA}

Universidad Nacional de La Plata, Argentina

ISSN: 1514-2590

Periodicidad: Semestral

vol.41,núm.1,2021

analecta@fcv.unlp.edu.ar

Recepción: 01 Diciembre 2020

Revisión: 03 Febrero 2021

Aprobación: 01 Marzo 2021

URL:

http://portal.amelica.org/ameli/jatsRepo/25/25198400 $1 /$ index.html

DOI:https://doi.org/10.24215/15142590eo53
Glycoprotein C induced a specific IgG response in serum. However, it failed to prevent entry of the virus into the lung or the appearance of clinical signs, which were variable among different treatment groups. Entry of the virus into the lung was delayed in all immunized groups. The viral titer was significantly lower when adjuvants were used, as well as in the intranasally immunized groups compared to the control group.

Therefore, glycoprotein $\mathrm{C}$ induced partial protection against the challenge with the virus, depending on the adjuvantand theroute of immunization.

Keywords: Equid alphaherpesvirus 1, glycoprotein C, immune response, BALB/cmice. 


\section{Introducción}

El alfaherpesvirus equino 1 (EHV-1) produce infección respiratoria, desórdenes neurológicos, abortos y síndrome neonatal en miembros de la familia Equidae, lo que provoca un significativo impacto económico negativo en la producción equina mundial. Debido a que la infección respiratoria natural no induce una respuesta inmune duradera, los animales permanecen susceptibles a reinfecciones durante su vida (Allen \& Bryans, 1986). La protección inmunológica de los caballos depende de la cooperación de la respuesta inmune humoral y celular específica, y se requiere de la presencia de anticuerpos (Ac) con actividad neutralizante para bloquear las partículas virales infecciosas libres y de linfocitos $\mathrm{T}$ citotóxicos para lisar las células infectadas (Wagner et al., 2015). La infección con EHV-1 causa, inicialmente, una respuesta inmune local en el epitelio nasofaríngeo que constituye el sitio primario de replicación, seguida de una respuesta inmune sistémica y de tipo celular. Sin embargo, la inmunidad protectora contra posteriores infecciones persiste solo entre 3 y 6 meses (Kydd et al., 2006). La inmunidad de mucosa, local y específica inducida por EHV-1 es relevante debido a que podría prevenir la entrada del virus a través de la membrana basal del epitelio nasofaríngeo y la inducción de viremia asociada a leucocitos mononucleares (Kydd et al., 2006; Vandekerckhove et al., 2010). Luego de la infección, el virus entra en estado de latencia en los linfocitos T CD8+y en el ganglio del nervio trigémino. La reactivación se produce cuando los animales se encuentran en situaciones estresantes, tales como transporte, medidas de manejo, tratamientos con corticoides o prácticas deportivas exigentes. Estas reactivaciones son generalmente silentes y la viremia que continúa a las mismas puede conducir al virus hasta el útero preñado y causar aborto, o al sistema nervioso central y producir síndrome neurológico (Allen, 2006).

El efectivo control de la infección se basa en la práctica de la vacunación y en la implementación de medidas de manejo adecuadas que disminuyan el estrés y prevengan la reactivación de virus latente y la consecuente diseminación. Actualmente se encuentran disponibles vacunas atenuadas y vacunas inactivadas, aunque ninguna de ellas es capaz de prevenir la infección primaria y la reinfección. Si bien las vacunas atenuadas tienen el riesgo de reversión de la virulencia de las cepas utilizadas en su elaboración, pueden proteger a los caballos contra la enfermedad clínica. Sin embargo, no está clara su eficacia en la prevención de la viremia, el aborto y el síndrome neurológico. Las vacunas inactivadas no evitan la infección; no obstante, inducen la producción de Ac neutralizantes y reducen tanto la cantidad de partículas virales infecciosas como el periodo de eliminación viral desde la nasofaringe (Kydd et al., 2006; Ma et al., 2013; Minke et al., 2004).

Las glicoproteínas de la envoltura viral juegan un importante rol en la infectividad y patogenicidad del virus y constituyen los blancos principales del sistema inmune (Allen \& Bryans, 1986), por lo que son fuertes candidatas para el desarrollo de vacunas a subunidad efectivas. La glicoproteína C (gC) está involucrada en la unión a la célula hospedadora y contribuye significativamente en la penetración eficaz del virus y en la eficiente replicación en células primarias equinas. Sin embargo, no es imprescindible para el crecimiento del virus en cultivos celulares 
(Huemer et al., 1995; Osterrieder, 1999). Diferentes glicoproteínas han sido producidas mediante técnicas de biología molecular y evaluadas como inmunógenos, tanto en ratones (Guo et al., 1990; Osterrieder et al., 1995; Packiarajah et al., 1998; Ruitenberg et al., 1999; Tewari et al., 1994,1995; Zhang et al., 1998) como en el hospedador natural (Foote et al., 2005, 2006). En dichos estudios se analizaron diferentes estrategias de vacunación para prevenir la infección, utilizando una sola glicoproteína o combinaciones de ellas, aunque en la mayoría delos trabajos se utilizaronglicoproteínas sin purificar y vías parenterales de administración (Weerasinghe et al., 2006). Recientemente se estudió, en equinos, una proteína de fusión compuesta por la gC de EHV-1 y la IL-4, con el propósito de desencadenar la capacidad natural de los basófilos neonatales para secretar IL-4. De esta manera se evaluó la activación de células $\mathrm{B}$ y la producción de Ac contra la gC, y se determinó que los animales desafiados con el virus obtuvieron protección parcial (Wagner et al., 2015). Por otro lado, en trabajos previos se observó que al inmunizar ratones $\mathrm{BALB} / \mathrm{c}$ por vía intranasal (IN) con la glicoproteína $\mathrm{D}(\mathrm{gD})$ se estimuló la producción de IgA en el tracto respiratorio y se indujo protección ante el desafío viral (Fuentealba et al., 2019). Continuando con esta línea de investigaciones, el objetivo de este trabajo fue expresar la gC de EHV-1, involucrada en la adsorción viral a la célula hospedadora, utilizando el sistema de baculovirus. Se evaluó la respuesta inmune inducida por esta $\mathrm{gC}$ recombinante en ratones $\mathrm{BALB} / \mathrm{c}$ inmunizados, utilizando diferentes vías de administración y distintos adyuvantes.

\section{Materiales y métodos}

\section{Expresión de la glicoproteína C}

Se trabajó con la cepa argentina AR8 de EHV-1, aislada en el año 1996 a partir del hígado de un feto abortado proveniente de un establecimiento ubicado en el partido de Magdalena (Provincia de Buenos Aires, Argentina). La patogenicidad de esta cepa fue evaluada en el modelo ratón BALB/c, por lo que es utilizada como nuestra cepa de referencia en todos los estudios en los quese utilizó en dicho modelo (Zanuzzi et al., 2014; 2016).

Para este trabajo se infectaron células RK13 (Rabbit kidney cells) con multiplicidad de infección (MOI) de 1. Las células infectadas se colectaron cuando se observó el efecto citopático típico de herpesvirus, sin lisis completa. Se realizó la extracción del ADN viral utilizando el equipo comercial Wizard® Genomic DNA Purification (Promega, Madison, WI, USA), de acuerdo con las instrucciones del fabricante, y se cuantificó mediante espectrofotometría (Smart specTM 3000, BIORAD) por lectura de absorbanciaa26onm.

Se amplificó mediante PCR un fragmento de 1212 pares de bases correspondiente a la porción codificante del gen de la gC, sin la región transmembrana, adicionándole una cola de histidina en el extremo N-terminal y los sitios de restricción para NcoI y XmaJI. Se utilizaron los siguientes oligonucleótidos: 
Las reacciones se llevaron a cabo en un volumen final de $25 \mu \mathrm{l}$, utilizando 2,5 $\mu$ lde Taq buffer $10 \mathrm{X}$ con $\mathrm{KCl}, 1,5 \mu \mathrm{M}$ de $\mathrm{MgCl}_{2}, 0,2 \mathrm{mM}$ de cada dATP, dCTP, dGTP y dTTP (Promega, Estados Unidos), 2,5 U de Taq DNA polimerasa (Fermentas, Tecnolab, CABA, Argentina), 0,5 $\mu \mathrm{M}$ de cada oligonucleótido (gC- F y gC-R), 14,5 $\mu$ l de agua libre de nucleasas y $3 \mu \mathrm{l}$ de muestra. Se utilizó un termociclador Mastercycler Gradient (Eppendorf, Netheler - Hinz GmbH, Hamburg) con el siguiente programa: desnaturalización inicial de 5 minutos a $94{ }^{\circ} \mathrm{C}$, seguida de 25 ciclos de 30 segundos a $94^{\circ} \mathrm{C}$ para la desnaturalización, 30 segundos a $58^{\circ} \mathrm{C}$ para la hibridación, 90 segundos a $72{ }^{\circ} \mathrm{C}$ de extensión y una extensión final de 5 minutos a $72{ }^{\circ} \mathrm{C}$. Para verificar la correcta amplificación de la gC, se sembró el producto de PCR en un gel de agarosa (Biodynamics, CABA, Argentina) al 1 \% en buffer Tris borato EDTA (TBE) 0,5X, el marcador de PM de 100 pb (Promega)y secorrióa 100V durante60 minutos.

El producto obtenido fue purificado utilizando el kit Wizard SV Gel \& PCR Clean Up (Promega) y posteriormente clonado en el vector pCR2.1TOPO, utilizando el kit TOPO TA Cloning (Life Technologies Corporation, Carlsbad, CA, Estados Unidos) y siguiendo las instrucciones de los fabricantes. Luego fue digerido con las enzimas de restricción correspondientes a los sitios de restricción agregados en los oligonucleótidos. El fragmento obtenido fue insertado en el sitio de clonado múltiple del vector de transferencia pFastBacHTB entre los sitios NcoI y XbaI, y fue utilizado para transformar bacterias E. coli cepa DH1oBac (Invitrogen, CABA, Argentina). La utilización de esos sitios de restricción permitió que la gC quedara fusionada y en marco con una cola de histidinas, y bajo el control del promotor de poliedrina del virus de la poliedrosis nuclear múltiple Autographa califórnica (AcMNPV). Se recuperó el ADN bacmídico recombinante para gC y fue utilizado para transfectar células de insecto Spodoptera frugiperda (Sf-21 o Sf-9) (Gibco, Grand Island, NY, USA). Una vez obtenido el baculovirus recombinante se utilizó para infectar células High Five (Invitrogen) con una MOI de 1 DICC $50 \%$ /célula y evaluar la expresión de la gC. Se colectaron los extractos de células infectadas a las 24, 48, 72 y 96 horas posinfección y se analizaron en gel de poliacrilamida con dodecilsulfato sódico (SDS-PAGE) al $10 \%$ y por inmunoblot, de acuerdo con los procedimientos estándar.

Una vez determinado el tiempo de mayor expresión de la gC recombinante $(\mathrm{gCr})$, se colectaron las células High Five previamente infectadas con el baculovirus recombinante en frascos de cultivo de $75 \mathrm{~cm}^{2}$, se sometieron a 3 ciclos de lisis por congelado y descongelado, y la suspensión resultante fue clarificada mediante centrifugación. Los $5 \mathrm{ml}$ de sobrenadante obtenido fueron utilizados para la purificación de la $\mathrm{gCr}$ utilizando el método de inmovilización por afinidad a iones metálicos (IMAC), con resina NTA-Ni (Qiagen, Germantown, USA). La elusión se realizó utilizando buffers de fosfatos (5O $\left.\mathrm{mM} \mathrm{PO}_{4} \mathrm{H}_{2} \mathrm{Na}, 500 \mathrm{mM} \mathrm{NaCl}\right)$ de $\mathrm{pH}$ decreciente ( $\mathrm{pH}$ 6, $\mathrm{pH} 5 \mathrm{y} \mathrm{pH}$ 4). La purificación fue verificada mediante electroforesis SDS-PAGE e inmunoblot. 


\section{Diseño experimental con ratones}

Los procedimientos experimentales que se llevaron a cabo en este trabajo se realizaron de acuerdo con las normativas internacionales, y fueron aprobados y supervisados por el Comité Institucional de Cuidados y Uso de Animales de Laboratorio (CICUAL) de la Facultad de Ciencias Veterinarias de la UNLP (Exp.600-004104/08-001. Res. 129/09). Se utilizaron ratones $\mathrm{BALB} / \mathrm{c}$ hembras de cinco semanas de edad, las que se mantuvieron en un aislador con temperatura controlada de $22{ }^{\circ} \mathrm{C}$, con ciclos de luz y oscuridad de 12 horas y con administración de alimento y agua ad libitum. Los animales se dividieron en ocho grupos de 10 ratones cada uno (denominados grupos A hasta $\mathrm{H}$ ) y, desde el grupo A hasta el G, fueron anestesiados con isofluorano (Baxter Co., Deerfield, IL, USA) e inmediatamente inmunizados con la gCr utilizando diferentes vías de administración. Enlosgrupos A, CyEseutilizólavíaintraperitoneal(IP), en los grupos B, Dy F la vía subcutánea (SC), y en el grupo G la vía IN. Además, en los animales inmunizados por las vías IP y SC se utilizaron los adyuvantes Specol (10 ratones por cada vía) y Montanide (10 ratones por cada vía), según se indica en la Tabla 1. El Montanide ISA 206 es un aceite mineral que contiene ésteres del ácido octadecenoico y anhidromanitol, cuya formulación es agua en aceite en agua (Barnett et al., 1996). Estas emulsiones tienen una baja viscosidad y la habilidad de aumentar la respuesta inmune a corto y largo plazo, y generalmente inducen inmunidad humoral. El adyuvante Specol es una emulsión de aceite mineral suave del tipo agua en aceite, que se puede utilizar para los Ag de baja inmunogenicidad y se puede administrar con la misma eficacia por vía SCo IP (Stills, 2005).

Cadaratón recibió, con unintervalode 20 días, dos dosis de $50 \mu \operatorname{ldeg} \mathrm{Cr}(15$ $\mu \mathrm{g}$ de proteína por dosis) en solución tamponada de fosfatos (PBS). El grupo control (grupoH)recibióPBSsolamenteporvíaIN.

Tabla 1. Inoculación de ratones BALB/c con la gC recombinante de alfaherpesvirus equino 1 (EHV-1)

\begin{tabular}{cccc}
\hline Grupo & $\begin{array}{c}\text { Vía de } \\
\text { inmunización }\end{array}$ & Adyuvante & $\begin{array}{c}\text { Desafío (cepa } \\
\text { AR8) }\end{array}$ \\
\hline A & IP & Specol & X \\
B & SC & Specol & X \\
C & IP & Montanide & X \\
D & SC & Montanide & X \\
E & IP & NO & X \\
F & SC & NO & X \\
G & IN & NO & X \\
H & Grupo control sin inmunizar (recibió PBS) & X \\
\hline
\end{tabular}

IP: intraperitoneal; SC: subcutánea; IN: intranasal. Todos los grupos fueron desafiados con $\sim 10^{6,3}$ DICC $_{50} \% / 50$ uldelacepaAR8deEHV-1.

\section{Desafio viral y toma de muestras}

Se tomaron pools de sangre de los animales de cada grupo, mediante punción de la vena maxilar, a los diez días de cada inmunización y antes del desafío. Cada pool se centrifugóa $1000 \mathrm{~g}$ durante 15 minutosyluego se separó el suero 
para ser usado en los ensayos de ELISA y neutralización viral (NV). A los 20 días de la segunda inmunización los animales fueron anestesiados, como fue descripto previamente, y desafiados por vía IN con $\sim 10^{6,3}$ DICC $50 \% / 50 \mu \mathrm{l}$ de la cepa AR8 de EHV-1. Luego del desafío viral se controló diariamente la aparición de signos clínicos característicos y se registró el peso corporal de todos los animales. Entre los días uno y cuatro posdesafío (pd), dos animales por grupo fueron diariamente anestesiados y sangrados a blanco sin heparina para obtener suero, y con heparina $(20 \mathrm{UI} / \mathrm{ml})$ para realizar aislamiento viral (AV) (Galosi et al., 2004) y detección de ADN viral mediante PCR a partir del plasma rico en leucocitos. Los sueros se utilizaron para determinar la presencia de Ac mediante ELISA y mediante NV. Durante la necropsia se tomaron muestras del pulmón izquierdo, las que se procesaron y utilizaron para determinar la presencia de virus en celulares, y del pulmón derecho para estudios histológicos. Se mantuvieron dos ratones por grupo hasta los 90 días de iniciado el plan de inmunización, se controló el peso corporal y se sangraron a los días 60 y 90, cuando finalizó la experiencia.

\section{Detección de anticuerpos}

Las muestras de suero de todos los grupos fueron analizadas mediante ELISA para la detección de IgG e IgA, utilizando un antígeno soluble producido en células MDBK (Madin-Darby bovine kidney) infectadas con EHV-1. El antígeno control se preparó con células MDBK sin infectar (Fuentealba, 2012). Se determinó la dilución óptima de uso de los sueros y se utilizaron los Ac secundarios anti-IgG de ratón con peroxidasa y antiIgA de ratón producido en cabra y conjugado con peroxidasa (Southern Biotech, Birmingham, AL, Estados Unidos). Como solución de sustrato se utilizó ABTS (ácido 2,2'-azino-bis-[3-etilbenzotiazolin-6-sulfónico] (Sigma, Estados Unidos)) diluido en una mezcla constituida por $50 \mathrm{ml}$ de buffer citrato $0,1 \mathrm{M}$ y $50 \mathrm{ml}$ de fosfato de sodio o,2 M, más el agregado de $0,01 \%$ de $\mathrm{H}_{2} \mathrm{O}_{2}$. Se realizó la lectura de los valores de densidad óptica (DO) utilizando un filtro de $405 \mathrm{~nm}$ en un lector para microplacas (Thermo Scientific Multiskan FC, Vantaa, Finlandia). El punto de corte $(0,250)$ fue determinado como el valor correspondiente al doble de la lectura promedio de la DO de los sueros del grupo control $\mathrm{H}$ antes del desafío viral (Crowther, 2009).

Para la NV los sueros fueron inactivados a $56{ }^{\circ} \mathrm{C}$ durante 30 minutos y posteriormente diluidos en base dos (desde $1 / 2$ a 1/512) en microplacas (Greiner BIO-ONE, Carolina del Norte, Estados Unidos) e incubados durante 1 hora con $\sim 100$ DICC $_{50 \%}$ de EHV-1. Finalmente, se agregaron células RK13 $\left(3 \times 10^{5}\right.$ células/ml), las que se incubaron bajo atmósfera controlada de $\mathrm{CO}_{2}$ hasta las 72 horas, momento en que se realizó la lectura final. Cada suero se analizó por duplicado y el título neutralizante de cada uno se expresó como la inversa de la mayor dilución que protegió del efecto viralaunadelasdosunidades deprueba (Cunningham, 1971). 


\section{TitulacióndeinfectividadyPCR}

El pulmón izquierdo de cada animal fue procesado de acuerdo con los métodos estándar, homogeneizando con arena estéril y Medio Esencial Mínimo (MEM) y centrifugando a $8000 \mathrm{~g}$ durante 20 minutos. El sobrenadante se inoculó sobre monocapa de células RK13, en diluciones seriadas en base diez realizadas en MEM. El título viral expresado en log DICC $_{50} \% / \mathrm{ml}$ se calculó por observación del efecto citopático y se cuantificó aplicando el método de Reed y Muench a las 96 horas.

Parte de los homogenatos de pulmón y del plasma rico en leucocitos fueron procesados para extracción de ADN utilizando, en ambos casos, el equipo comercial Wizard $\AA$ Genomic DNA Purification. Por último, se realizó la detección mediante PCR.

\section{Estudios histopatológicos y de inmunohistoquímica (IHQ)}

Las muestras de pulmón derecho fueron fijadas en formol y procesadas utilizando las técnicas de rutina para estudios histológicos. Se obtuvieron cinco secciones de $3 \mu \mathrm{m}$ del pulmón de cada animal; algunas fueron coloreadas con hematoxilina y eosina y otras utilizadas para IHQ para detectar antígenos de EHV-1 siguiendo los protocolos previamente descriptos (Zanuzzi et al., 2014). Brevemente, los antígenos virales fueron detectados utilizando suero policlonal de conejo anti EHV-1 en una dilución de $1 / 1600$ en PBS más $0,1 \%$ de seroalbúmina bovina, y el Ac secundario anti-conejo EnVision $囚$ detection system $+\mathrm{HRP}$ (DakoCytomation, Carpinteria, CA, Estados Unidos). La reacción se reveló con el agregado de 3,3'diaminobenzidina (DAB) y se realizó una coloración nuclear de contraste con hematoxilina.

\section{Análisis estadístico}

Se analizaron los datos obtenidos de los pesos corporales de los animales, los valores de DO obtenidos mediante ELISA y los títulos virales en pulmón, mediante la prueba Shapiro-Wilk para verificar su distribución normal. Se utilizó ANOVA de una vía, con medidas repetidas en el tiempo, seguido por la prueba post hoc de Bonferroni para determinar posibles diferencias entre grupos.

\section{Resultados}

\section{Expresión de la glicoproteína $C$}

Seobtuvounbaculovirus recombinanteparala expresión delgen delagCsin la región transmembrana. Los resultados del inmunoblot mostraron que la gCr se expresó en forma antigénica detectable desde las 48 horas posinfección, con una concentración máxima a las 96 horas posinfección. Las células High Five infectadas con el baculovirus recombinante mostraron un patrón de bandas similar en todas las muestras tomadas a diferentes tiempos posinfección, observándose dos bandas de aproximadamente 60 kDay 55 kDa (Figura 1). Luego de la purificación con NTA-Ni, también se 
verificó mediante SDS-PAGE la presencia de las dos bandas específicas de $55 \mathrm{y}$ 60 kDa, aproximadamente. La cuantificación de la gCr purificada arrojó un valor de $300 \mu \mathrm{g} / \mathrm{ml}$.
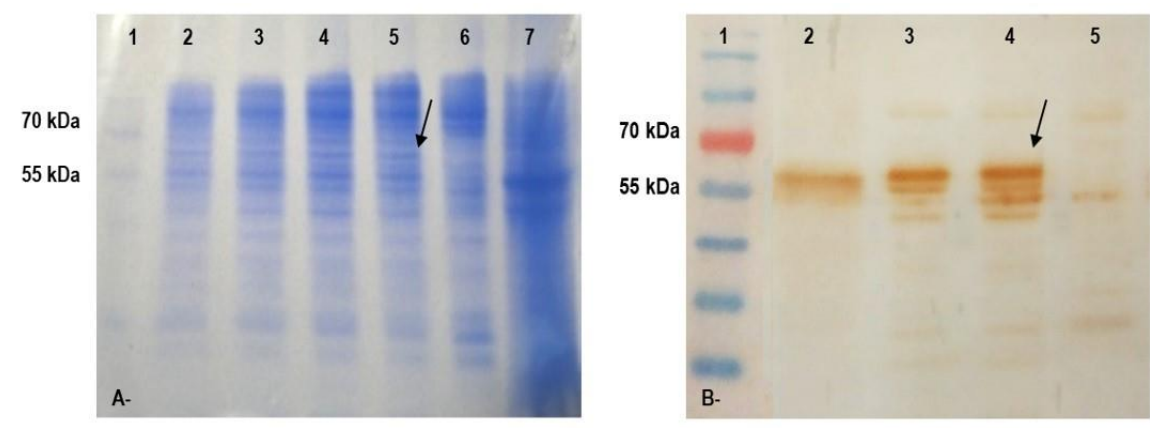

Figura 1. A. SDS-PAGE de células High Five infectadas con baculovirus recombinante. Línea 1: Marcador de PM (PAGE- Ruler, Fermentas); líneas 2 a 5: células infectadas colectadas a las 24, 48, 72 y 96 horas posinfección; línea 6: control de células sin infectar; línea 7: control de virus. B. Técnica de inmunoblot: células High Five infectadas con baculovirus recombinante y analizadas utilizando un suero policlonal anti-EHV-1. Línea 1: Marcador de PM (PAGE-Ruler, Fermentas); línea 2: control de virus; líneas 3 y 4: células infectadas colectadas a las 72 y 96 horas posinfección; línea 5: control de células sin infectar. Las flechas indican las bandas correspondientes a la gCr.

\section{Evaluación clínica}

Los ocho grupos de ratones, los inmunizados con la gCr utilizando diferentes vías, y el grupo control, fueron desafiados con la cepa AR8 de EHV-1. Los pesos corporales antes del desafío se distribuyeron normalmente y no mostraron diferencias entre los grupos ( $\mathrm{p}>0,05)$. Después del desafío, todos los animales del grupo control (grupo $\mathrm{H}$ ) presentaron signos clínicos característicos, tales como depresión general, pelo hirsuto, disnea y disminución del peso corporal desde el día dos pd, mientras que en la mayoría de los animales de los grupos inmunizados los signos se registraron desde el tercer día pd. Se observaron diferencias entre los grupos, respecto del peso, teniendo en cuenta el uso o no de adyuvantes, y los días analizados $(\mathrm{p}<0,05)$. El peso de los animales comenzó a recuperarse el día 10 pd para todos los grupos. El grupo $\mathrm{E}$ ( $\mathrm{gCr}$, vía IP) fue el único grupo inmunizado en el cual todos los ratones mostraron disminución del peso corporal al primer día pd, y comenzaron a recuperarlo el día 10, al igual que el resto delos grupos (Figura 2).

\section{Detección de anticuerpos}

Las muestras de suero fueron analizadas mediante ELISA para la detección de IgG e IgA. Se observó que la gCr no indujo la producción de IgG en ausencia de adyuvancia (grupos E, F y G) a los diez días de la primera inmunización, y tampoco fue detectada luego de la segunda inmunización; resultados similares mostraron los grupos $\mathrm{A}(\mathrm{gC}+\mathrm{Specol}$, vía IP) y $\mathrm{C}$ ( $\mathrm{gC}+$ Montanide, vía IP). En el caso de los grupos B ( $\mathrm{gC}+\mathrm{Specol}$, vía $\mathrm{SC}$ ) y $\mathrm{D}$ (gC+Montanide, vía $\mathrm{SC}$ ) se detectó la producción de IgG luego de la primera inmunización, pero no se observó un aumento en la DO entre los valores hallados a los días diez y 30. Luego del desafío viral se observó un aumento significativo en el valor de DO paratodos los gruposinmunizados 
con gCr combinada con algún adyuvante, y fue detectable hasta el final del experimento (día 90). En el caso de los grupos inmunizados sin adyuvante y utilizando las vías IP y SC (grupos Ey F) se observó un leve aumento en el valor deDO, elquedisminuyórápidamente(Figura3).

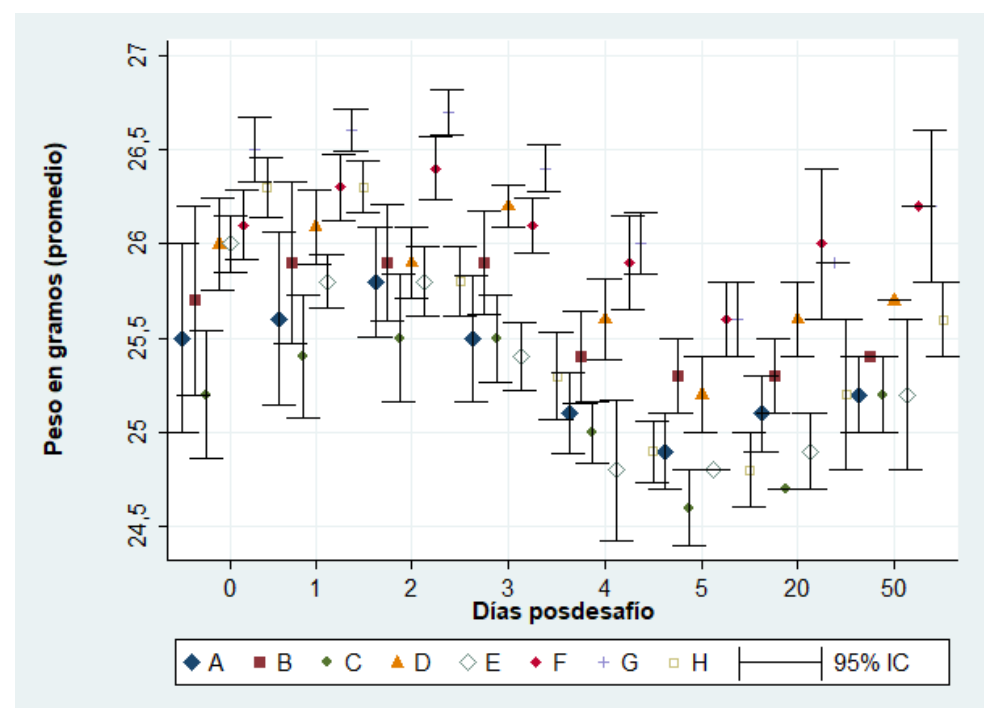

Figura 2. Peso de ratones BALB/c $(n=10$ en cada grupo $)$ inmunizados congCrydesafiados con $\sim 10^{6,3}$ $\mathrm{DICC}_{50} \% / 50 \mu \mathrm{l}$ de la cepa AR8 de EHV-1. Cada punto representa el promedio de $\mathrm{n}=10$ en el día o y 1 posdesafío (días 40-41 posinmunización), n-2 en los días 2-4 posdesafío (días 42-44 posinmunización) y $\mathrm{n}=2$ los días 5,20 y 50 después del desafío (días 45, 60 y 90 después de la inmunización). Grupos de trabajo: A (gCr con Specol por vía intraperitoneal); B (gCr con Specol por vía subcutánea); $\mathrm{C}$ ( $\mathrm{gCr}$ con Montanide por vía intraperitoneal); $\mathrm{D}$ ( $\mathrm{gCr}$ con Montanide por vía subcutánea); $\mathrm{E}$ (gCr por vía intraperitoneal); $\mathrm{F}$ (gCr por vía subcutánea); $\mathrm{G}$ (gCr por vía intranasal); $\mathrm{H}$ (controlsininocular).

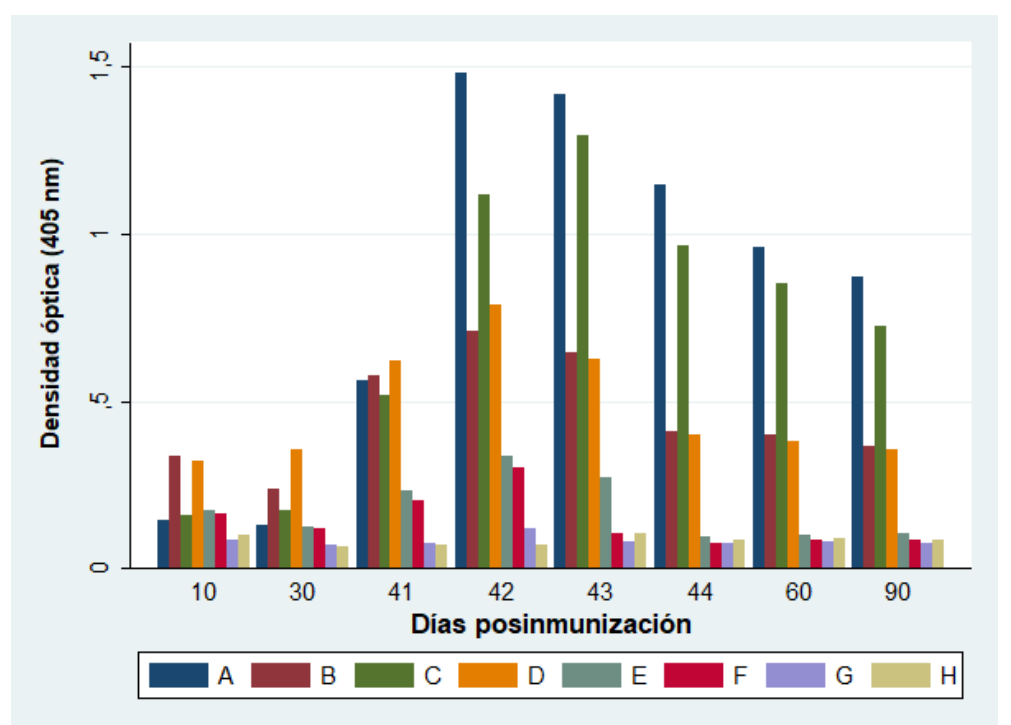

Figura 3. Técnica de ELISA: respuesta de anticuerpos específicos (IgG) en ratones BALB/c inmunizados y desafiados al día 40 con $\sim 10^{6,3}$ DICC $_{50} \% / 50 \mu$ ldela cepa AR8 de EHV-1. Los ratones se inmunizaron dos veces, con 20 días de diferencia, con diferentes formulaciones de gCr de EHV-1 purificada. Los primeros dos valores corresponden a la DO medida para el pool de sueros de cada grupo con $n=10$ (días 10 y 30). Los restantes valores corresponden al promedio de la DO para $\mathrm{n}=2$ de cada grupo (días 41, 42, 43, 44, 60 y 90). Grupos de trabajo: A (gCr con Specol por vía intraperitoneal); B ( $\mathrm{gCr}$ con Specol por vía subcutánea); C ( $\mathrm{gCr}$ con Montanide por vía intraperitoneal); $\mathrm{D}$ ( $\mathrm{gCr}$ con Montanide por vía subcutánea); $\mathrm{E}$ (gCr por vía intraperitoneal); $\mathrm{F}$ (gCr por vía subcutánea); $\mathrm{G}$ ( $\mathrm{gCr}$ porvíaintranasal); $\mathrm{H}$ (controlsininocular). 
En el análisis realizado para determinar qué grupos eran disímiles estadísticamente, se observaron diferencias mínimas significativas $(\mathrm{p}<0,05)$ entre los grupos A y C respecto de los grupos restantes (B y D-H). De la misma manera, se observaron diferencias significativas entre los grupos B y D, que utilizaron la misma vía de inmunización y diferentes adyuvantes, con respecto a los grupos restantes. En el caso de los grupos E, F y G, en los cuales no se utilizaron adyuvantes, se observaron diferencias significativas con respecto a lo observado en los grupos inmunizados utilizando adyuvantes (A-D). Nosedetectó IgAen sueroderatones deningunodelos grupos.

Se determinó que los Ac inducidos neutralizaron la actividad viral. En los grupos $\mathrm{B}$ ( $\mathrm{gC}+$ Specol, vía $\mathrm{SC})$ y $\mathrm{D}$ ( $\mathrm{gC}+$ Montanide, vía $\mathrm{SC}$ ) se detectaron Ac neutralizantes luego de la segunda inmunización, y se incrementaron al día tres pd. Para los grupos A ( $\mathrm{gCr}+\mathrm{Specol}$, vía IP) y C (gCr+Montanide, vía IP) se observó, luego del desafío, un alto título de Ac, los que se mantuvieron detectables hasta la finalización del ensayo. En los grupos en los que no se utilizaron adyuvantes, el título de Ac fue menor. Para el grupo control el resultado fue siempre negativo (Tabla 2).

Tabla 2. Técnica de neutralización viral.

\begin{tabular}{ccccccccc}
\hline Días & \multicolumn{8}{c}{ Grupos } \\
\cline { 2 - 9 } & A & B & C & D & E & F & G & H \\
\hline 30 & - & 16 & - & 16 & - & - & - & - \\
41 & 64 & 16 & 64 & 16 & - & - & - & - \\
42 & 128 & 16 & 64 & 16 & 8 & 8 & - & - \\
43 & 128 & 32 & 64 & 32 & 8 & 8 & - & - \\
44 & 64 & 32 & 32 & 32 & - & - & 4 & -
\end{tabular}

Anticuerpos neutralizantes detectados en ratones inmunizados dos veces, con un intervalo de 20 días, con diferentes formulaciones de $\mathrm{gC}$ recombinante de alfaherpesvirus equino 1 (EHV-1) purificada, y posteriormente desafiados al día 40 con $\sim 10^{6,3} \mathrm{DICC}_{50} \% / 50 \mu \mathrm{l}$ de la cepa AR8 de EHV-1. El primer valor corresponde al título de anticuerpos neutralizantes del pool de sueros de cada grupo con $n=10$ (día 30). Los restantes valores corresponden al promedio del título para $\mathrm{n}=\mathbf{2}$ de cada grupo (días 41, $42,43,44)$. Los títulos son expresados comola inversa dela mayor dilución de suero que protegió del efecto viral a una de las dos unidades de prueba. Grupos de trabajo: A (gCr con Specol por vía intraperitoneal); $\mathrm{B}$ ( $\mathrm{gCr}$ con Specol por vía subcutánea); $\mathrm{C}$ ( $\mathrm{gCr}$ con Montanide por vía intraperitoneal); $\mathrm{D}$ (gCr con Montanide por vía subcutánea); $\mathrm{E}$ (gCr por vía intraperitoneal); $\mathrm{F}$ (gCr porvíasubcutánea); G(gCrporvíaintranasal); H(control).

\section{AislamientoviralyPCR}

El AV y la detección de $\mathrm{ADN}$ a partir de pulmones y de plasma rico en leucocitos, fueron positivos para todos los animales del grupo control (grupo $\mathrm{H})$ desde el día uno hasta el día cuatro pd.

Paralos grupos E(gCr, vía IP), F (gCr, vía SC) y G (gCr, vía IN) la detección de ADN y el AV fueron positivos desde el día dos hasta el día cuatro pd a partir de pulmón y de plasma rico en leucocitos. El título viral detectado en pulmón para los grupos mencionados fue significativamente menor que los hallados para elgrupocontrol $(\mathrm{p}<0,05)$. 
En los grupos restantes (A, B, C y D) solamente se recuperó virus de pulmón al segundo y tercer día pd, con títulos virales similares y significativamente menores que para el grupo control $(\mathrm{H})(\mathrm{p}<0,05)$ (Figura 4). Además, se detectó ADN viral en las mismas muestras y en plasma rico en leucocitos de los grupos $\mathrm{A}, \mathrm{B}$ y $\mathrm{D}$ a los días uno y dos pd y del grupo $\mathrm{C}$ al día dos pd.

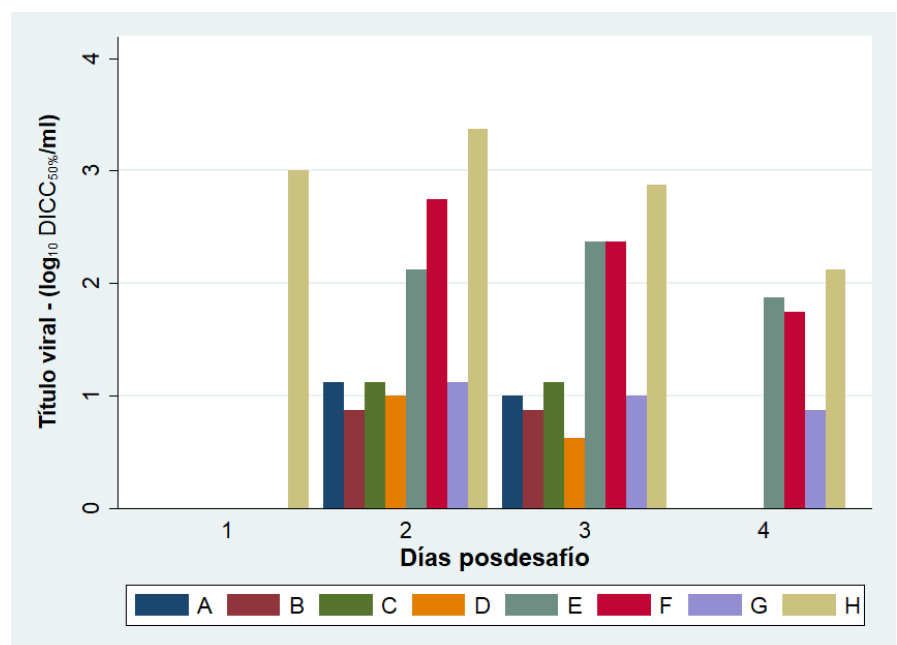

Figura 4. Títulos virales en pulmón de ratones BALB/cinmunizados y desafiados al día 40 con $\sim 10^{6,3}$ $\mathrm{DICC}_{50} \% / 50 \mu \mathrm{l}$ dela cepa AR8 deEHV-1. Se tomaron muestras depulmón de cada ratón alos días 41, 42, 43 y 44 después de la primera inmunización (días 1, 2, 3 y 4 después del desafío). Los títulos virales de los pulmones se calcularon mediante el método de Reed y Muench y se expresaron comolog1o DICC $50 \% / \mathrm{ml}$. Los datos representan el título medio obtenido de dos pulmones procesados individualmente. Grupos de trabajo: A ( $\mathrm{gCr}$ con Specol por vía intraperitoneal); B ( $\mathrm{gCr}$ con Specol por vía subcutánea); $\mathrm{C}$ (gCr con Montanide por vía intraperitoneal); $\mathrm{D}$ ( $\mathrm{gCr}$ con Montanide por vía subcutánea); $\mathrm{E}$ (gCr por vía intraperitoneal); $\mathrm{F}$ (gCr por vía subcutánea); $\mathrm{G}$ (gCr por vía intranasal); $\mathrm{H}$ (control sininocular).

\section{Estudios histopatológicos y por inmunohistoquímica (IHQ)}

Los pulmones de los animales de todos los grupos, incluido el grupo control (grupo H), presentaron cambios histopatológicos característicos de la infección con EHV-1. Se observó un proceso inflamatorio leve, caracterizado por un infiltrado con células mononucleares y escasos neutrófilos alrededor de las vías respiratorias y vasos sanguíneos. Se observaron células sincitiales, descamación y cuerpos de inclusión intranucleares eosinofílicos en los epitelios bronquiolares. En el grupo control (grupo H) se observó necrosis bronquiolar severa desde el día uno pd, mientras que en los grupos inmunizados los cambios se observaron desde el día dos pd. Mediante IHQ se detectaron antígenos virales en el epitelio bronquial y bronquiolar en las muestras del grupo control en el día uno pd y en los restantes grupos desde el día dos pd.

\section{Discusión y conclusiones}

La infección por el EHV-1 causa un importante impacto en la salud y en la producción equina en el mundo. A pesar de que existe una amplia 
variedad de vacunas contra esta virosis, su efectividad continúa en discusión (Lunn et al., 2009). Las vacunas comerciales disponibles actualmente, muchas de las cuales están elaboradas a partir de virus inactivado, han demostrado reducir en un $75 \%$ la incidencia de tormentas de abortos en yeguas preñadas, cuando se aplican junto a buenas prácticas de manejo. Sin embargo, los animales continúan siendo susceptibles a la reinfección (Kydd etal., 2006).

Para patógenos como el EHV-1, que generalmente infecta los animales en los primeros años de vida, el plan de vacunación utilizado con las vacunas comerciales no parece ser eficaz para prevenir la primoinfección de los potrillos $\mathrm{y}$, en consecuencia, el establecimiento de una infección latente en una etapa temprana de la vida (Equine Herpesvirus (Rhinopneumonitis); Vaccination guidelines; AAEP, 2020). El desarrollo de vacunas que estimulen una eficiente respuesta inmunológica a la infección por el EHV-1 continúa siendo prioritario. Se ha estudiado el potencial inmunogénico de vacunas elaboradas a partir de diferentes glicoproteínas de envoltura de EHV-1, utilizando tanto el modelo murino (Guo et al., 1990; Osterrieder et al., 1995; Ruitenberg et al., 1999; Tewari et al., 1994, 1995; Zhang et al., 1998, 2000) como el hospedador natural (Foote et al., 2005,2006; Wagneretal., 2016)de enfermedad respiratoria.

La gC deEHV-1, comose mencionó, es la proteína involucrada en la unión al receptor celular y en la entrada a la misma (Huemer et al., 1995) y se comprobó que esta glicoproteína es inmunogénica tanto en el modelo ratón como en el hospedador natural (Packiarajah et al., 1998, Wagner et al., 2015).

En este trabajo se expresó la gC de EHV-1 mediante la obtención de un baculovirus recombinante y se verificó la expresión de dicha glicoproteína mediante SDS-PAGE, detectándose la presencia de un producto de aproximadamente $60 \mathrm{kDa}$. Este resultado fue confirmado mediante inmunoblot, con el que también se detectó un producto adicional de aproximadamente $55 \mathrm{kDa}$. Ambos productos posiblemente representan distintos estados de procesamiento postraduccional (glicosilaciones) y fueron observados durante todo el ensayo de expresión en diferentes concentraciones. Con estos resultados, se descarta que hubiese degradación de la proteína durante su producción y se demuestra la capacidad inmunogénica de la glicoproteína obtenida. Nuestros resultados contrastan con los que reportan otros autores (Stokes et al., 1996), quienes al expresar la gC truncada en baculovirus detectaron la presencia de una banda de $80 \mathrm{kDa}$ a las 24 horas pi y varias bandas de distinto tamaño luego de las 48 horas pi, probablemente debido a la degradación parcial de la proteína producida.

En nuestros ensayos experimentales llevados a cabo en ratones inmunizados con la gCr, la aparición de signos clínicos frente al desafío con el virus fue observada más tardíamente en comparación con el grupo control sin inmunizar. El peso corporal en la mayoría de los grupos inmunizados comenzó a disminuir uno o dos días después que lo observado en el grupo control, con la excepción de un grupo inmunizado sin adyuvante por vía IP. Sin embargo, a partir del día diez pd el peso se recuperó en todos los grupos estudiados. Resultados similares fueron 
descritos por Stokes etal. (1996) al inmunizar ratones con la gCtruncada, purificada y sin purificar y utilizando la vía IP en ambos casos. Por el contrario, nuestros resultados difieren de los reportados por otros autores (Packiarajah et al., 1998), quienes observaron que los animales inmunizados con gC completa y sin purificar (células de insecto infectadas con baculovirus recombinante para gC) por vía IM, mostraron severos signosclínicos, aligualqueelgrupo control, al ser desafiados.

En este trabajo, evaluamos dos adyuvantes alternativos de aceite mineral comercial: Montanide ISA 206 (Ganne et al., 1994) y Specol (Stills et al., 2005). En los ratones inmunizados por vía SC con la gCr y ambos adyuvantes, se estimuló la producción de IgG a partir del décimo día posinmunización; sin embargo, en los grupos inmunizados utilizando la vía IP se detectó IgG luego del desafío viral. En todos los grupos, estos Ac persistieron hasta el final de la experiencia (día 90 posinmunización). En los animales inmunizados sin adyuvantes (grupos E, F y G), la gCr no indujo la producción de IgG en suero luego de cada inmunización, resultado que también se había observado para la gD (Fuentealba et al., 2019). Sin embargo, al tratarse de pools de muestras por grupo, no se descarta un efecto de dilución de sueros positivos a IgG con el suero de los animales negativos. De modo similar a un trabajo en el que se utilizaron cuatro dosis de la gC sin purificar (Packiarajah et al., 1998), observamos bajos niveles de Ac. Sin embargo, estos autores demostraron que cuando la gC fue utilizada en combinación con otras glicoproteínas, comogB ogD, se obtuvo un alto nivel de Ac, con capacidad neutralizante ycon títulos moderados. En coincidencia, Stokes et al. (1996) también detectaron bajo título de Ac neutralizantes, a pesar de que demostraron que el epitope correspondiente está presente en la glicoproteína obtenida. En nuestro trabajo la producción de IgG detectada mediante ELISA se observó en los mismos grupos en los cuales se detectaron Ac neutralizantes. A diferencia de lo observado para la gD (Fuentealba et al., 2019), el adyuvante Montanide ISA 206 demostró una alta eficacia para inducir respuesta de Ac neutralizantes cuando se utilizó la vía $\mathrm{SC}$, respuesta que puede explicarse por diferencias en la efectividad de la combinación de adyuvantes con determinadas proteínas (Stills, 2005).

En nuestro ensayo, se recuperó virus de los pulmones de todos los grupos de ratones inmunizados, a partir del segundo día pd, a diferencia de lo ocurrido en el grupo control en el que se recuperaron partículas virales desde el primer día pd. Además, se observaron títulos virales bajos y similares entre los grupos inmunizados utilizando algún adyuvante, y sólo en estos grupos el virus fue eliminado de los pulmones antes que en el grupocontrol.

Otras de las glicoproteínas de envoltura de EHV-1 es la gD, la cual proporciona altos niveles de protección al ser evaluada en el modelo murino (Whalley et al., 2002). Cuando evaluamos la gD como inmunógeno, se observó que al inmunizar por vía IN y desafiar a los animales con el virus, este no llegaba al pulmón (Fuentealba et al., 2019), por lo que la vía utilizada resultaba efectiva para proteger a los animales frente al desafío viral. Contrariamente a lo ocurrido con la gD, luego de la inmunización con la gCr por la misma vía de inoculación, los resultados fueron diferentes, debido a que se recuperó virus de los pulmones. Sin embargo, el título 
viral obtenido fue menor que en los encontrados en el grupo control.

Por otro lado, nuestros hallazgos con la $\mathrm{gCr}$, al inmunizar por vías parenterales, son similares a los reportados por Stokes et al. (1996), quienes observaron una reducción del título viral en los pulmones de los animales inmunizados por vía IP con la gC purificada y sin purificar. Estos autores, además, detectaron menor título viral que en los controles cuando analizaron los cornetes nasales de los animales en los que utilizaron la gC purificada. Por el contrario, otros autores (Packiarajah et al., 1998), no recuperaron virus en los animales inmunizados por vía IM con cuatro dosis de células Sf-9 expresando la gC y postularon que la inmunización con esta glicoproteína impidió la llegada del virus a los pulmones debido a la producción de Ac neutralizantes. Sin embargo, en sus experiencias, no evaluaron la presencia del EHV-1 a los días uno y dos pd, por lo que esta hipótesis no logró ser confirmada.

Los estudios histológicos realizados en este trabajo confirmaron los resultados hallados por aislamiento viral y PCR. Se observaron lesiones compatibles con la infección en las muestras de pulmón de aquellos animales donde fue posibleaislar elvirus luego del desafío.

Por lo tanto, este trabajo demuestra que la gCr estimula la producción de Ac solamente cuando se utiliza combinada con algún adyuvante y es administrada por vías parenterales, promoviendo la protección parcial frente al desafío viral. Contrariamente a lo observado con la gD (Fuentealba et al., 2019), la inmunización a través de la vía IN no fue capaz de proteger a los animales contra la infección con EHV-1. Probablemente la gCr requiere de un adyuvante apropiado para la formulación al utilizar la vía IN de inmunización.

Sin embargo, y teniendo en cuenta los resultados obtenidos por el grupo de trabajo con la gD (Fuentealba et al., 2019) y los observados en el presente trabajo, se plantea como posible estrategia inmunológica alternativa el uso de una primera dosis de la gD administrada por vía IN y una segunda dosis con la $\mathrm{gCr}$ administrada por vía parenteral, lo que favorecería, probablemente, una respuesta inmune más eficiente y la protección frente al desafío viral. De esta manera, si la protección inducida por la gD es parcial y logra interactuar con la barrera de la mucosa nasofaríngea, entonces un objetivo secundario sería asegurar una respuesta inmunológica sistémica, que podría ser provista por la gCr al utilizar una vía diferente a la IN. Por lo tanto, es necesario continuar evaluando el posible uso de la gCr obtenida en este trabajo para lograr una inmunización efectiva contra el EHV-1.

\section{Agradecimientos}

Agradecemos al Dr. Miguel Ayala (Laboratorio de Animales de Experimentación, Facultad de Ciencias Veterinarias, UNLP) por su asistencia profesional y a los técnicos Rubén Mario y Claudio Leguizamón por su colaboración en el procesamiento de las muestras y cuidado de los animales. Este estudio fue financiado por la Academia Nacional de Agronomía y Veterinaria, la Agencia Nacional de Promoción Científica y Tecnológica (PICT 2011-1123), la Secretaría de Ciencia y Técnica de la UNLP y la Comisión de Investigaciones Científicas de la Provincia de Buenos Aires. 


\section{Declaración de conflicto de intereses}

No existe conflicto de intereses, incluyendo entre estos últimos las relaciones financieras, personales o de otro tipo con otras personas u organizaciones que pudieran influir de manera inapropiada en este trabajo.

\section{Bibliografía}

Allen GP, Bryans JT. Molecular epizootiology, pathogenesis and prophylaxis of equine herpesvirus-1 infections. En: Pandey R.1986. Progress in Veterinary Microbiology and Immunology. Basilea, Karger, pp.78-144.

Allen GP. 2006. Antemortem detection of latent infection with neuropathogenic strains of equine herpesvirus-1 in horses. American Journal of Veterinary Research. 67(8):1401-5.

https://doi.org/10.2460/ajvr.67.8.1401

American Association of Equine Practitioners (AAEP). 2020. Equine Herpesvirus (Rhinopneumonitis); Vaccination guidelines. [En línea] Disponible en: https://aaep.org/guidelines/vaccination-guidelines/riskbased-vaccination-g uidelines/equine-herpesvirus-rhinopneumonitis [Consultado 20/7/2020].

Barnett PV, Pullen L, Williams L, Doel TR. 1996. International Bank for foot-andmouth disease vaccine: assessment of Montanide ISA 25 and ISA 206, two commercially available oil adjuvants. Vaccine. 14(13): 1187-98. https://doi.org/ 10.1016/s0264-410x(96)00055-2

Crowther JR. 2009. The ELISA Guidebook. $2^{\circ}$ Ed. Vienna, Austria. Humana Press. Cunningham HC. 1971. Virología Práctica. Zaragoza, España, Editorial Acribia.

Foote CE, Love DN, Gilkerson JR, Rota J, Trevor-Jones P, Ruitenberg KM, Wellington JE, Whalley JM. 2005. Serum antibody responses to equine herpesvirus 1 glycoprotein $\mathrm{D}$ in horses, pregnant mares and young foals. Veterinary Immunology and Immunopathology. 105(1-2):47-57. https://doi.org/10.1016/ j.vetimm.2004.12.012

Foote CE, Raidal SL, Pecenpetelovska G, Wellington JE, Whalley JM. 2006. Inoculation of mares and very young foals with EHV-1 glycoproteins D and B reduces virus shedding following respiratory challenge with EHV-1. Veterinary Immunology and Immunopathology. 111(1-2):97-108.

https://doi.org/10.1016/j.vetimm.2006.01.012

Fuentealba NA. 2012. Herpesvirus equino 1: estudio de la expresión de proteínas antigénicas y evaluación de la respuesta inmune inducida. Tesis de doctorado en Ciencias Veterinarias, Facultad de Ciencias Veterinarias, Universidad Nacional de La Plata.

Fuentealba NA, Sguazza GH, Zanuzzi CN, Bravi ME, Scrochi MR, Valera AR, Corva SG, Gimeno EJ, Pecoraro MR, Galosi CM. 2019. Immunoprotective response induced by recombinant glycoprotein $\mathrm{D}$ in the BALB/c respiratory mouse model of Equid alphaherpesvirus 1 infection. Revista Argentina de Microbiología. 51(2):119-29. https://doi.org/10.1016/j.ram.2018.05.004

Galosi CM, Barbeito CG, Vila Roza MV, Cid de la Paz V, Ayala MA, Corva SG, Etcheverrigaray ME, Gimeno EJ. 2004. Argentine strain of equine herpesvirus 1 isolated from an aborted foetus shows slow virulence in mouse respiratory and abortion models. Veterinary Microbiology. 103(1-2):1-12.

https://doi.org/10.10 16/j.vetmic.2004.07.015 
Ganne V,Eloit M, Laval A, Adam M, Trouve G. 1994.Enhancement of the efficacy of a replication-defective adenovirus-vectored vaccine by the addition of oil adjuvants. Vaccine. 12:1190-6.

https://doi.org/10.1016/0264-410x(94)90242-9

Guo PX, Goebel S, Perkus ME, Taylor J, Norton E, Allen G, Languet B, Desmettre P, Paoletti E. 1990. Coexpression by vaccinia virus recombinants of equine herpesvirus 1 glycoproteins gp13 and gp14 results in potentiated immunity. Journal of Virology. 64(5):2399-406.

https://doi.org/10.1128/JVI.64.5.2399-24 06.1990

Huemer HP, Nowotny N, Crabb BS, Meyer H, Hübert PH. 1995. gp13 (EHV$\mathrm{gC}$ ): a complement receptor induced by equine herpesviruses. Virus Research. 37(2):113-26. https://doi.org/10.1016/0168-1702(95)00027-n

Kydd JH, Townsend HGG, Hannant D. 2006. The equine immune response to equine herpesvirus-1: the virus and its vaccines. Veterinary Immunology and Immunopathology. 111(1-2):15-30.

https://doi.org/10.1016/j.vetimm.2006.01.005

Lunn DP, Davis-Poynter N, Flaminio MJ, Horohov DW, Osterrieder K, Pusterla N, Townsend HG. 2009. Equineherpesvirus-1 consensus statement. Journal of Veterinary Internal Medicine. 23(3):450-61. https://doi.org/10.1111/j.1939-16 76.2009.0304.x

Ma G, Azab W, Osterrieder N. 2013. Equine herpesviruses type 1 (EHV-1) and 4 (EHV-4) masters of co-evolution and a constant threat to equids and beyond. Veterinary Microbiology. 167(1-2):123-34.

https://doi.org/10.1016/j.vetmic.20 13.06.018

Minke JM, Audonnet JC, Fischer L. 2004. Equine viral vaccines: the past, present and future. Veterinary Research. 35(4):425-43.

https://doi.org/10.1051/vetres:2004019

Osterrieder N, Wagner R, Brandmüller C, Schmidt P, Wolf H, Kaaden OR. 1995. Protection against EHV-1 challenge infection in the murine model after vaccination with various formulations of recombinant glycoprotein gp14 (gB). Virology. 208(2):500-10. https://doi.org/10.1006/viro.1995.1181

Osterrieder N. 1999. Construction and characterization of an equine herpesvirus 1 glycoprotein C negative mutant. Virus Research. 59:165-77. https://doi.org/10. 1016/s0168-1702(98)o0134-8

Packiarajah P, Walker C, Gilkerson J, Whalley JM, Love DN. 1998. Immune responses and protective efficacy of recombinant baculovirus-expressed glycoproteins of equine herpesvirus $1(\mathrm{EHV}-1) \mathrm{gB}, \mathrm{gC}$ and $\mathrm{gD}$ alone or in combinations in BALB/ c mice. Veterinary Microbiology. 61(4):261-78. https://doi.org/10.1016/s0378-1135(98)o0189-8

Ruitenberg KM, Walker C, Wellington JE, Love DN, Whalley JM. 1999. ADNmediated immunization with glycoprotein D of equine herpesvirus $1(\mathrm{EHV}-1)$ in a murine model of EHV-1 respiratory infection. Vaccine. 17(3):237-44. https:// doi.org/10.1016/So264-410X(98)00192-3

Stills HF. 2005. Adjuvants and antibody production: dispelling the myths associated with Freund's complete and other adjuvants. ILAR Journal. 46(3):280-93. https://doi.org/10.1093/ilar.46.3.280

Stokes A, Alber DG, Cameron RS, Marshall RN, Allen GP, Killington RA. 1996. The production of a truncated form of baculovirus expressed EHV-1 glycoprotein $\mathrm{C}$ and its role in protection of $\mathrm{C}_{3} \mathrm{H}(\mathrm{H}-2 \mathrm{Kk})$ mice against virus challenge. 
Virus Research. 44(2): 97-109.

https://doi.org/10.1016/0168-1702(96)01339-1

Tewari D, Nair SV, De Ungria MC, Lawrence GL, Hayden M, Love DN, Field HJ, Whalley JM. 1995. Immunization with glycoprotein C of equine herpesvirus1 is associated with accelerated virus clearance in a murine model. Archives of Virology. 140(4):789-97. https://doi.org/10.1007/BF01309967

Tewari D, Whalley JM, Love DN, Field HJ. 1994. Characterization of immune responses to baculovirus expressed equine herpesvirus type 1 glycoproteins D and $\mathrm{H}$ in a murine model. Journal of General Virology. 75(Pt 7):1735-41. https://do i.org/10.1099/0022-1317-75-7-1735

Vandekerckhove A, Glorieux S, Gryspeerdt AC, Steukers L,Duchateau L, Osterrieder N, Van de Walle GR, Nauwynck HJ. 2010. Replication kinetics of neurovirulent versus non-neurovirulent equine herpesvirus type 1 strains in equine nasal mucosal explants. The Journal of General Virology. 91(Pt 8):2019-28.https://d oi.org/10.1099/vir.0.019257-0

Wagner B, Goodman LB, Babasyan S, Freer H, Torsteinsdót-tir S, Svansson V, Björnsdóttir S, Perkin GA. 2015. Antibody and cellular immune responses of naïve mares to repeated vaccination with an inactivated equine herpesvirus vaccine. Vaccine.33(42):5588-97.

https://doi.org/10.1016/j.vaccine.2015.09.009

Whalley JM, Love DN. 2002. Improved vaccine strategies for management of equine herpesvirus. Report of Rural Industries Research and Development Corporation, Sydney. Disponible en https://www.agrifutures.com.au/wpcontent/uploads/publications/02-111.pdf [Consultado 10/3/2020]

Weerasinghe CU, Learmonth GS, Gilkerson JR, Foote CE, Wellington JE, Whalley JM. 2006. Equine herpesvirus 1 glycoprotein D expressed in E. coli provides partial protection against equine herpesvirus infection in mice and elicits virus-neutralizing antibodies in the horse. Veterinary Immunology and Immunopathology. 111(1-2):59-66.

https://doi.org/10.1016/j.vetimm.2006.01.009

Zanuzzi C, Scrochi M, Fuentealba N, Nishida F, Portiansky E, Muglia C, Gimeno E, Barbeito C, Galosi C. 2014. Effects of equid herpesvirus 1 (EHV-1) AR8 and HH1 strains on BALB-c mice. Archives of Virology. 159(1):141-5.

https://doi.org/10.1007/s00705-013-1782-8

Zanuzzi CN, Bravi ME, Scrochi MR, Nishida F, Fuentealba NA, Diessler ME, Sguazza HG, Muglia CI, Gimeno EJ, Portiansky EL, Barbeito CG, Galosi CM. 2016. Microvascular lesions and changes in cell proliferation and death, and cytokine expression in the placentas of mice experimentally infected with equid herpesvirus 1. Research in Veterinary Science. 109:121-8. https://doi.org/10.1016/j.rvsc.20 16.09.005

Zhang Y, Smith PM, Tarbet EB, Osterrieder N, Jennings SR, O'Callaghan DJ. 1998. Protective immunity against equine herpesvirus type-1(EHV-1) infection in mice induced by recombinant EHV-1 gD. Virus Research. 56(1):11-24. https://doi.org/10.1016/So168-1702(98)ooo54-9

Zhang Y, Smith PM, Jennings SR, O'Callaghan DJ. 2000. Quantitation of virusspecific classes of antibodies following immunization of mice with attenuated equine herpesvirus 1 and viral glycoprotein D. Virology. 268(2):482-92. https://doi.org/10.1006/viro.2000.0197 